\title{
Power Consumption Optimization of Location Management in PCS Network
}

\author{
Sumanta Kumar Deb \\ Dept. of Computer Science \& Engineering \\ Institute of Engineering \& Management \\ Kolkata, India
}

\author{
Sauvik Bal \\ Dept. of Computer Science \& Engineering \\ Institute of Engineering \& Management \\ Kolkata, India
}

\begin{abstract}
In this paper, we develop an approach to optimize power consumption using solar energy and also develop an approach for storing power as backup power resource. The following aspects of this paper are 1) location management based on location update (LU) and paging method by considering the location area (LA) architecture in personal communication service (PCS) networks. 2) Try to reduce location management cost by utilizing global natural solar energy resource by solar energy harvester and 3) create a backup storage for solar energy during day—off situation.
\end{abstract}

\section{General Terms}

PCS network, Power consumption, location area.

\section{Keywords}

PCS, VLR, HLR, UE, LU

\section{INTRODUCTION}

PCS stands for personal communication services. In PCS network, location of User Equipment (UE) is recorded by the mechanism called Location Management (LM) [1]. In LM, there are two operations, Location Update (LU) and Paging [1]. By using Location Update (LU) user equipment can update their locations and by paging, UE dynamically updates UEs location through network management [3]. When incoming call is connected, by using paging, actual situation area of UE is pointed out. The total cost of LM depends upon the parameters like bandwidth usage, battery power, computing capacity etc. Bandwidth usage refers the data volume which passes over either the wired medium or the wireless medium. Battery power indicates the consumption of battery which is needed to active the UE during the location management operation. Computing capacity indicates memory size of UE [1]. If the memory size is large then more power will be consumed. Frequently LUs generate high LU cost. In PCS network, to manage the location, PCS network is divided into small areas called Registration Areas. Each registration area consists of an aggregation of cells. To track the user equipment this registration area is called Location Area (LA). Similarly in 3G network, it is called Routing Area (RA) and in advanced 4G Long time evaluation (LTE) network, these registration area is called tracking area (TA) [1]. Always it should maintain two database, home and visited database. The profile information of UEs is stored in home database. In home database this information are stored like, the UE's identification, services subscribed, authentication parameters, current location and so forth. The home database is called Home Location Register (HLR) and the visited database is called Visitor Location Register (VLR) [1][2].
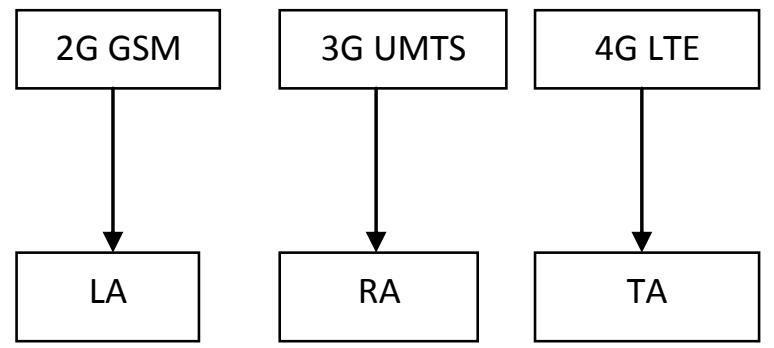

Fig 1: Registration area of 2G, 3G, 4G

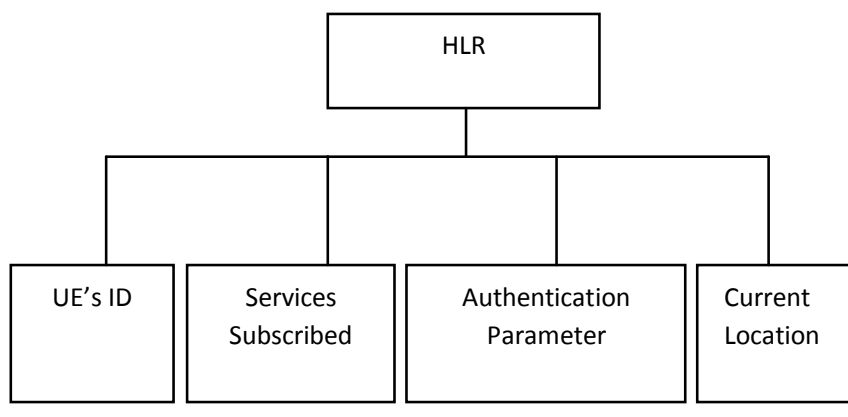

Fig 2: Several parameters of HLR

In static mode, the UE moves from an old LA into a new LA, The UE initiates an LU toward the new VLR that provides the new LA, which reports its new location to the HLR. After receiving the report, the HLR instructs the old VLR that provides the old LA to delete the record of the UE and simultaneously transfers the UE's subscriber data to the new VLR. Under the static scheme, all the UEs have paging areas of the same size that is the size of an LA [1]. The static scheme is not cost effective, because it neglects the mobility characteristics of individual UE. In PCS network, the UEs with low mobility, frequent LUs are necessary to reduce the paging cost that reduces the signaling cost, whereas UE devices with high mobility, infrequent LUs are necessary to reduce the LU cost that reduces the signaling cost. But if a UE has no incoming call then in such a situation, no LU is needed. Therefore, a cost effective LM scheme should be dynamic and can adapt to different mobility patterns. 


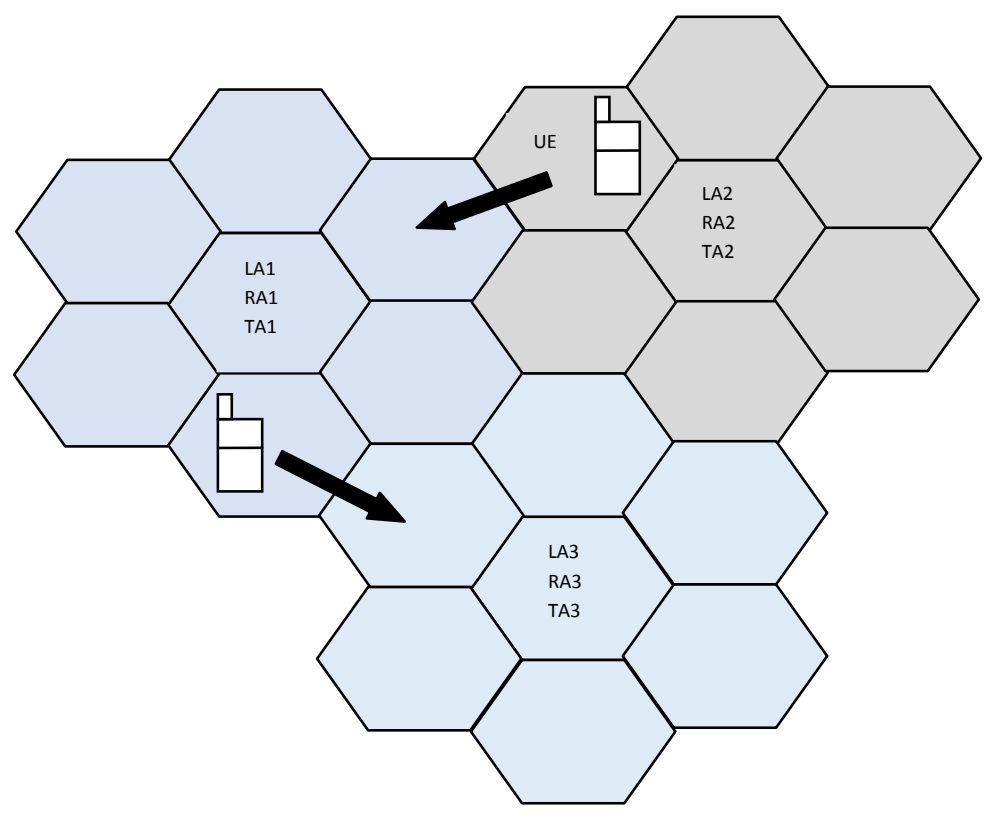

Fig 3: Block diagram of LA/RA/TA in PCS network

\section{DESCRIPTION}

1. Each HLR contains MSC which maintains one database that contains the information about the UE [2].

2. The UE moves from an old LA into a new LA, the UE initiates An LU toward the new VLR [1].

3. The new VLR (VLR1) serves the new LA to UE.

4. The UE reports its new location to the HLR.

5. The HLR instructs the old VLR (VLR1) that serves the old LA to delete the record of the UE.

6. Simultaneously transfers the UE's subscriber data to the VLR1.

7. Under the static scheme all the UEs have paging areas of the same size that is the size of a LA.

8. Again, the UE moves from an old LA of VLR1 into a new LA of VLR2, the UE initiates An LU toward the new VLR (VLR2).

9. The new VLR (VLR2) serves the new LA to UE.

10. The UE reports its new location to the old VLR (VLR1).

11. The VLR1 reports UE's new location to the HLR.

12. The HLR instructs the old VLR (VLR1) that serves the old LA to delete the record of the UE.

13. Simultaneously transfers the UE's subscriber data to the VLR1.

14. Similarly, VLR1 transfers the UE's subscriber data to the VLR2.

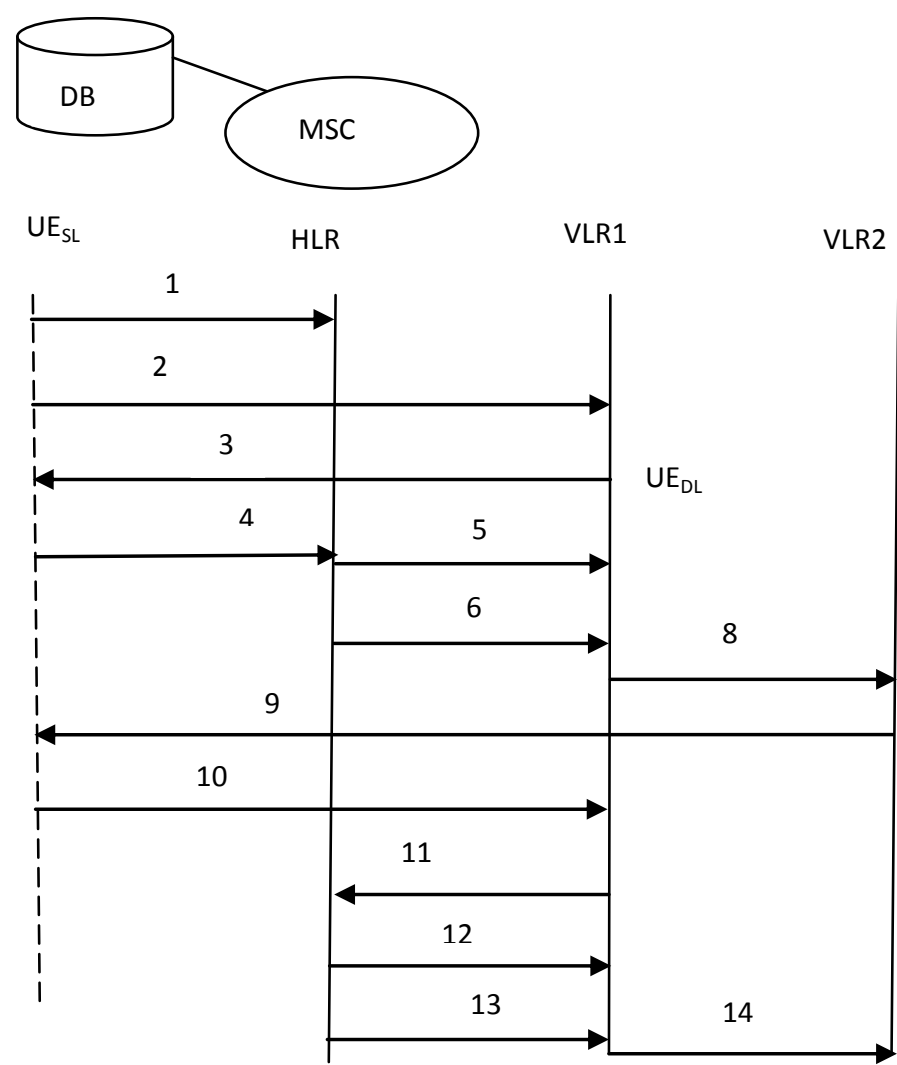

Fig 4: Location Management (LM) of UE

Next, we describe about the concept of Solar Energy Harvesting technique, which is used to solve the problem of power consumption [8]. Solar provides an excellent source of energy for wireless systems that have no access to fixed power. Solar energy is abundant, present in all. On average, the amount of solar energy is falling onto a square meter at the equator of planet earth is $1000 \mathrm{w} / \mathrm{m} 2$. This number varies wildly depending on circumstances and location but illustrates the point that solar energy can provide significant amounts of power for outdoor wireless applications.

A typical solar energy scavenging system is represented by the five main circuit system blocks shown in Fig.4 consists of a free energy source such as a small photovoltaic cell which is exposed to either direct sunlight or even indoor lighting. These photovoltaic cells are capable of generating over $50 \mathrm{~mW}$ of electrical power per square $\mathrm{cm}$ of area in peak sunlight and up to $100 \mu \mathrm{W}$ of electrical power in indoor lighting. However, the electrical energy must be collected in a very specific manner using an energy harvesting circuit (Figure 6) that can efficiently collect this low voltage energy and convert it into an usable form, which can be used to continually charge a storage device [8]. As the power generated by the solar cell that will vary dramatically with the ambient lighting conditions. A rechargeable storage device such as a battery or supercap (block three in Figure 4) is required to provide continuous power when the ambient light is no longer available. In turn, the storage device, whether a battery or a supercap, combined with a simple step-down DC/DC converter (fourth block in Figure 4, which is usually not needed) can power downstream electronics while it is continually recharged [8]. 


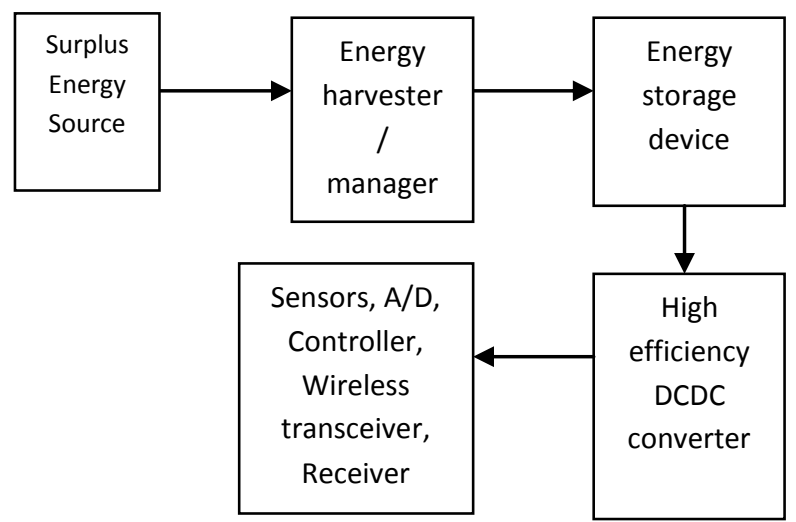

Fig 4: Block diagram solar energy storing circuit

\section{PROPOSED WORK}

In this paper, we focus on solar power consumption of the UE, thereby reducing the overall Location Management cost [1]. We have developed a cellular architecture. A base station has been implemented at the center of each cell. We have implemented the Ant Colony Optimization Algorithm for the reduction of power consumption of the UE, thereby optimizing the overall Location Management Cost. Ant Colony Based Scheduling Algorithm (ACB-SA) plays an important role [7]. This algorithm is used to solve the problem of Energy -Efficient Coverage (EEC). In this section, we introduce in detail our proposed algorithm for reduction of power consumption with the help of Solar Energy Harvesting. In ACB-SA, we first check the number of point of Interest (PoIs) that can be covered by each sensor with position information. We then initialize the pheromone filed with these values. By doing this, there was a strong possibility that ants can select the sensors that cover more PoIs at the beginning. Therefore, this initialization method help the ACB-SA find the optimal solution more systematically by using the sensors in the WSNs time progressed [5]. But a problem may arise if there is huge traffic in the path, which the ants are following to reach the destination. Then, they have to change and follow another path where there is no traffic.

In this case, power consumption will be increased; therefore the overall Location Management Cost will be also increased. We have to track our UE accurately as well as we also have to reduce our power consumption.

To track the exact location of UE, we have to first find the Tracking area List (TAL) [1][5]. Next, we have to find the tracking area at which UE is residing, then we can track the exact location of UE, and to solve the power supply problem, we have to provide a constant supply of energy, and this can be done by using Solar Energy Harvesting process [8]. At day light, UE receives energy from Sun, and can also use the power that is residing within it, that it took from the power supply earlier. The Solar Energy Harvester Manager stores the energy in the Energy storage device. The Energy storage device divides the energy into two ways. One is going to the current usage and another is going for back up purpose. When the current usage level is zero, immediately it takes energy from the back -up energy. This phenomenon will work as uninterrupted power supply (UPS) (Figure 6). In this way, we can track the exact location of UE, no matter how many searching process we have to do, power can be saved, and also the overall Location Management cost is minimized.

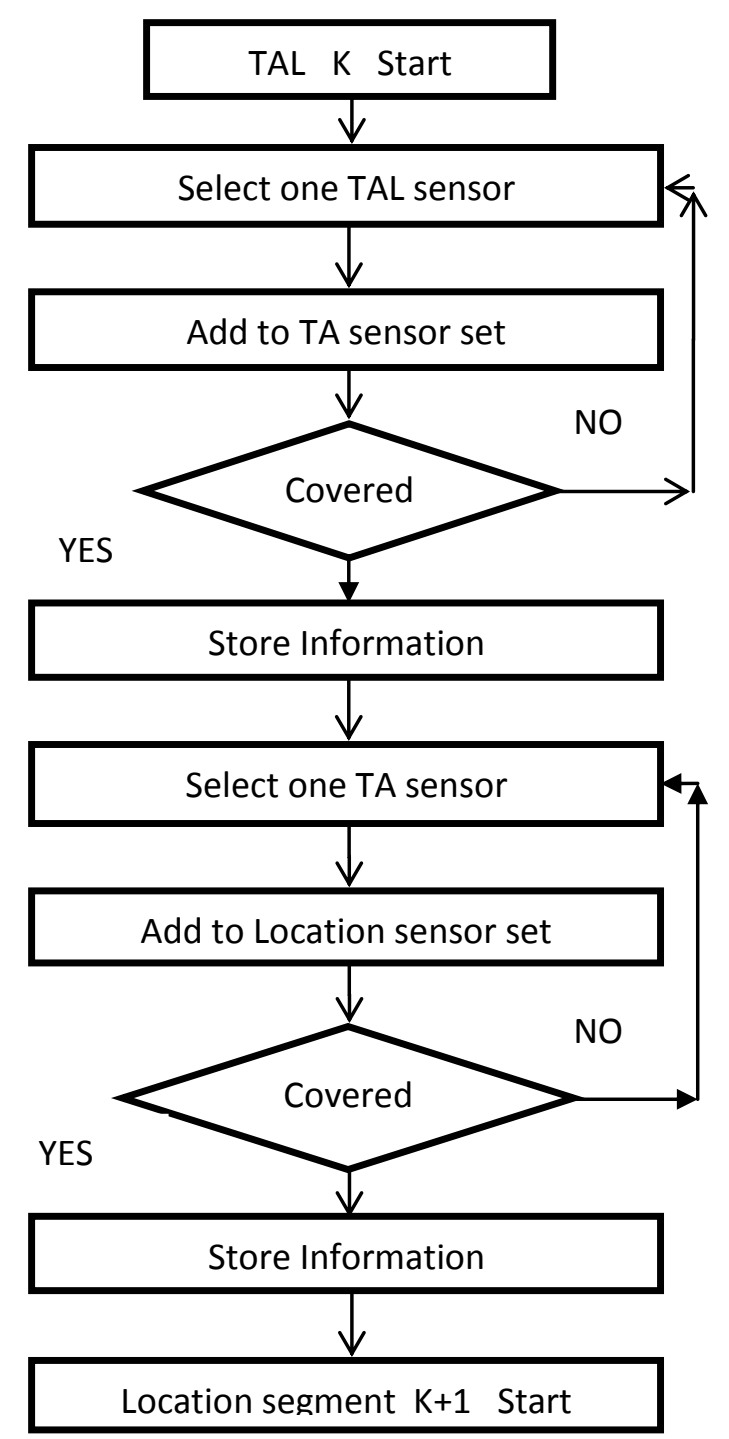

Fig 6: Flow chart of movement of UE

\section{ALGORITHM}

Set Parameter (Un, TALn, P, E) //Un - No. of user; TALn No. of TAL; P - how much time an user stays at a certain place; E - exact value of the time, say $10 \mathrm{hrs} . / /$

Initialize(C, Ts)

TS 0; // TS - time slot

While termination condition does not meet

do

// new time slot start

TS TS+1

nt 0 ;

Initialize (Iuser, Llist)

While $\mathrm{nt}<=$ TALn do

//New TA start

nt $n t+1$; 
While it does not meet do

//User k's travel Start

i TAs Select;

Iuser,k(i) 1

end while

//Cut the TA list, and search the exact location

Sort (Llist(1),.....,Llist(2Un));

$\operatorname{Llist}(U n)=\operatorname{Cut}(\operatorname{Llist}(1), \ldots \ldots, \operatorname{Llist}(U n))$;

CbestLlist(1);

Update (P)

end while

return $\mathrm{C}$, Ts;

We have to use solar energy for power saving in parallel.

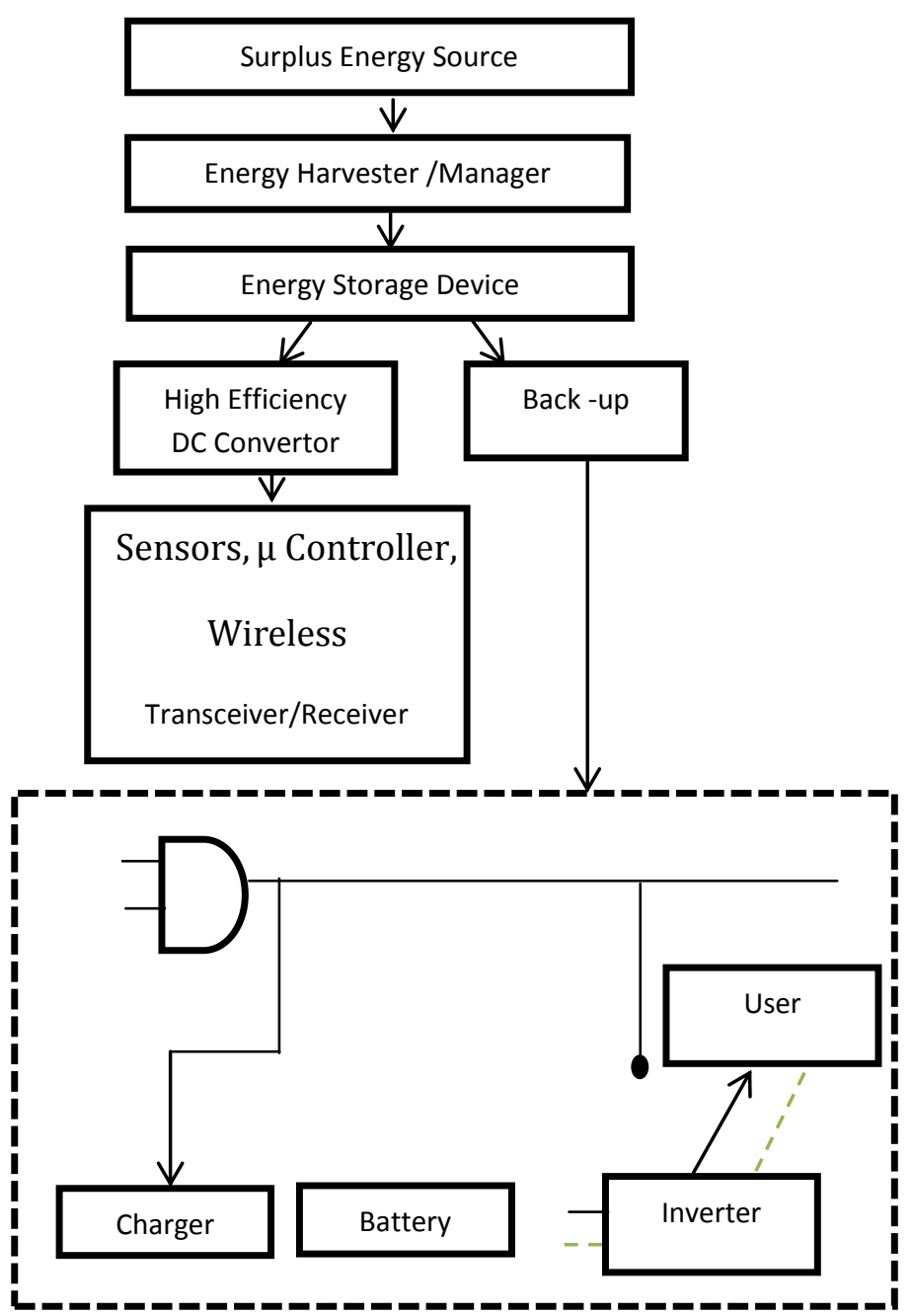

Fig 6: Flow chart of converting solar energy and storing solar energy

\section{CONCLUSION}

In this paper, we solved the problem of reduction of power consumption in PCS network using Solar Energy Harvester which is a more realistic approach where solar energy is used in two modes, as running usage mode and as a backup mode. To achieve this, we introduced a technique, where the UE can change the existing path and can follow another path in case of huge traffic on their way, and simultaneously power can also be saved using Solar Energy Harvester, so that the overall Location Management cost can also get reduced and location management can be done efficiently.

\section{ACKNOWLEDGMENTS}

This research paper is made possible through the help and support from everyone like our parents, faculties, colleagues. So we would like to thank all of them. Also we would like to thank our respected director sir, IEM, Kolkata for his support and encouragement to do this research work.

\section{REFERENCES}

[1] Xian Wang, Member, IEEE, Xianfu Lei, Member, IEEE, Pingzhi Fan, Senior Member, IEEE, Rose Qingyang $\mathrm{Hu}$, Senior Member, IEEE, and Shi-Jinn Horng; "Cost Analysis of Movement-Based Location Management in PCS Networks: An Embedded Markov Chain Approach - IEEE ,14”.

[2] "Cellular radio-telecommunications intersystem operations," EIA/TIA, Dallas, TX, USA, EIA/TIA Tech. Rep. IS-41, Rev. B, 1991.

[3] I. F. Akyildiz, J. S. M. Ho, and Y.-B. Lin, "Movementbased location update and selective paging for PCS networks," IEEE/ACM Trans. Netw.,vol. 4, no. 4, pp. 612-638, Aug. 1996.

[4] Y. Fang, "Movement-based mobility management and trade off analysis for wireless mobile networks," IEEE Trans. Comput., vol. 52, no. 6, pp. 791-803, Jun. 2003.

[5] K. Li, "Cost analysis and minimization of movementbased location management schemes in wireless communication networks: A renewal process approach," Wireless Netw., vol. 17, no. 4, pp. 1014-1053, May 2011.

[6] J.-Y. Heo, J.-M. Hong, and Y.-K. Cho, "EARQ: Energy aware routing for real-time and reliable communication in wireless industrial sensor networks," IEEE Trans. Ind. Inf., vol. 5, no. 1, pp. 3-11, Feb. 2009.

[7] Ant-Colony-Based Scheduling Algorithm for EnergyEfficient Coverage of WSN Joon-Woo Lee, Student Member, IEEE, and Ju-Jang Lee, Fellow, IEEE

[8] Jeff Gruetter, Sr. Product Marketing Engineer, Power Products, Linear Technology "SOLAR ENERGY HARVESTING” OCTOBER 2010. 